\title{
Determination of thresholds for minimally important difference and clinically important response on the functional outcomes of sleep questionnaire short version in adults with narcolepsy or obstructive sleep apnea
}

\author{
Terri E. Weaver $^{1}$ (D) $\cdot$ Diane M. Menno ${ }^{2} \cdot{\text { Morgan } \text { Bron }^{3} \cdot \text { Ross D. Crosby }}^{4,5} \cdot$ Susan Morris ${ }^{3} \cdot$ Susan D. Mathias ${ }^{5}$
}

Received: 22 September 2020 / Revised: 18 November 2020 / Accepted: 8 December 2020 / Published online: 4 January 2021

(C) The Author(s) 2021

\begin{abstract}
Purpose This study estimated thresholds for clinically important responses and minimally important differences for two indicators of improvement for the 10-item version of the functional outcomes of sleep questionnaire (FOSQ-10).

Methods Participants with excessive daytime sleepiness with narcolepsy or obstructive sleep apnea received 12 weeks of solriamfetol treatment. Participants completed the FOSQ-10 and other patient-reported outcome measures, including the single-item patient global impression of change (PGI-C) assessment. Clinicians completed the single-item clinician global impression of change (CGI-C) for each participant. Data from the two studies were analyzed separately, both without regard to treatment assignment. In total, 690 participants (47\% female, mean age 48 years, 77\% Caucasian, 91\% from North America) were enrolled. Two clinically important changes, defined as a minimally important difference and a clinically important response, were determined using distribution and anchor-based analyses. A receiver operating characteristic analysis was used to determine the optimal FOSQ-10 change threshold.

Results Spearman correlations between change in FOSQ-10 scores and PGI-C and CGI-C were -0.57 and -0.49 for participants with narcolepsy and -0.42 and -0.37 for participants with obstructive sleep apnea. Receiver operating characteristic analysis suggested minimally important difference and clinically important response estimates of 1.7 and 2.5 and 1.8 and 2.2 points in narcolepsy and obstructive sleep apnea, respectively.

Conclusions Minimally important difference and clinically important response estimates for the FOSQ-10 for adults with excessive daytime sleepiness in narcolepsy or obstructive sleep apnea will be helpful for interpreting changes over time and defining a clinical responder.

ClinicalTrials.gov identifiers NCT02348593 (first submitted January 15, 2015) and NCT02348606 (first submitted January 15 ,
\end{abstract} 2015)

Keywords Quality of life · Treatment outcome · Clinically important difference · Clinical significance $\cdot$ Hypersomnolence disorders $\cdot$ Sleep-disordered breathing

Classification 10.150 Sleep disordered breathing therapy $\cdot 20.400$ Narcolepsy $\cdot 100.300$ Daytime sleepiness

Terri E. Weaver

teweaver@uic.edu

College of Nursing, Department of Biobehavioral Nursing Science and College of Medicine, Department of Medicine, Division of Pulmonary, Critical Care, Sleep \& Allergy, University of Illinois at Chicago, 845 South Damen Avenue MC 802, Chicago, IL 60612, USA
Jazz Pharmaceuticals, Philadelphia, PA, USA

Jazz Pharmaceuticals, Palo Alto, CA, USA

Sanford Center for Bio-Behavioral Research, Fargo, ND, USA

5 Health Outcomes Solutions, Winter Park, FL, USA 


\section{Introduction}

Excessive daytime sleepiness (EDS) can be caused by a variety of conditions, including narcolepsy, obesity, sleep apnea, and other chronic diseases [1]. EDS is a clinical hallmark of narcolepsy and obstructive sleep apnea (OSA) [2, 3]. Often the first symptom observed, EDS occurs in $100 \%$ of those with narcolepsy [2]. Among those with OSA, the prevalence of EDS has been shown to vary by gender [4] and OSA severity [5]. Among patients treated with continuous positive airway pressure (CPAP) therapy, residual EDS has been reported by 9 to $22 \%$, depending on average duration of nightly CPAP use [6]. Those with EDS associated with OSA or narcolepsy experience fatigue, impaired work performance, emotional worry, decreased focus, impaired personal and family relationships, and falling asleep while driving [7,8]. Additionally, EDS can impair functionality and may result in poorer social engagement [9].

The Functional Outcomes of Sleep Questionnaire (FOSQ) is a 30-item, disease-specific, quality-of-life questionnaire designed to assess the impact of EDS on activities of everyday living and the extent to which these activities are improved by effective treatment [9]. An abbreviated 10-item version of the questionnaire (FOSQ-10) was developed as an alternative for use in clinical trials and everyday clinical practice [10]. The reliability, validity, and responsiveness of the FOSQ-10 have been previously demonstrated [10], but estimates of how much change is clinically meaningful have not been previously established.

Establishing thresholds that characterize a meaningful change to patients can help clinicians interpret results from patient-reported outcome measures and may inform clinical decisions regarding patient care [11, 12]. These thresholds can also help researchers interpret the clinical significance of changes within a study and estimate the necessary sample size for randomized trials [11].

We sought to identify two thresholds for change for the FOSQ-10. The first, the minimally important difference (MID), is defined as the smallest change that a patient is able to identify. The second, the clinically important response (CIR), indicates a change that represents a relevant treatment benefit [13]. By establishing these thresholds, we intend to improve the understanding of how to interpret increments of change within the FOSQ-10.

\section{Methods}

\section{Study population and data sources}

Data were analyzed from participants with EDS associated with narcolepsy or OSA who were enrolled in one of two phase 3, multicenter, randomized, double-blind, 12-week studies $[14,15]$ of solriamfetol, a dopamine and norepinephrine reuptake inhibitor approved in the USA and European Union for these disorders. Full details of these studies' methods, participant populations, and primary efficacy and safety outcomes have been reported previously $[14,15]$. Both studies were approved by institutional review board or ethics committees at each site, and all participants provided written informed consent $[14,15]$. Most inclusion and exclusion criteria were similar: both trials included adult participants (aged 18-75 years) with a body mass index from 18 to $<45 \mathrm{~kg} / \mathrm{m}^{2}$ and examined the safety and efficacy of solriamfetol for EDS. However, some inclusion criteria differed slightly; one trial involved participants with OSA, while the other enrolled those with narcolepsy without moderate to severe OSA. Additionally, while inclusion criteria regarding the Epworth Sleepiness Scale (ESS) were the same in the two trials, there were slightly different requirements for baseline sleep latency based on the Maintenance of Wakefulness Test (MWT): patients in the OSA trial were included if their sleep latency was less than $30 \mathrm{~min}$, whereas patients in the narcolepsy trial were included if their sleep latency was less than $25 \mathrm{~min}$. For the OSA study, participants had OSA diagnosed according to International Classification of Sleep Disorders, $3 r d$ edition (ICSD-3) criteria and current or prior use of a primary OSA therapy, including a CPAP machine, oral appliance, or surgical intervention [15]. Participants in the narcolepsy study were diagnosed according to ICSD-3 criteria or Diagnostic and Statistical Manual of Mental Disorders, 5th edition [14] criteria. Treatment groups were also slightly different in the two studies: while each trial included groups receiving $75 \mathrm{mg}, 150 \mathrm{mg}$, and $300 \mathrm{mg}$ (as well as a placebo group), the OSA trial also included an additional dose comparator of $37.5 \mathrm{mg}$ that the narcolepsy trial did not.

Participants completed the MWT and ESS. The MWT is administered by trained clinicians and measures the ability to stay awake for a defined period of time, requiring participants to try to stay awake as long as possible while seated in a comfortable chair in a darkened room [16]. The ESS is a self-administered questionnaire that asks participants to rate their likelihood of dozing off or falling asleep during eight different activities [17]. Participants also completed the FOSQ-10 and the single-item Patient Global Impression of Change (PGI-C) rating. Clinicians completed a single-item Clinician Global Impression of Change (CGI-C) rating for each participant. Both impression of change ratings (PGI-C and CGI-C) assessed the change in the participant's condition from baseline to week 12 using a 7-point scale ranging from 1 (very much improved) to 7 (very much worse). In contrast to the original 30-item FOSQ, the FOSQ-10, which was originally developed for application in the clinical setting, is an abbreviated 10-item version that can be readily used in clinical practice and research. The FOSQ-10 is scored to provide a total score containing items related to general productivity, 
activity level, vigilance, social outcomes, and intimacy. It has previously been shown to have strong psychometric properties and to perform similarly to the FOSQ-30, with high internal consistency, reliability, and effect sizes and pre- and posttreatment differences that are highly correlated with the 30 item version [10].

\section{Statistical analysis}

Data from the two studies (NCT02348593 and NCT02348606) were analyzed separately, both without regard to randomization group. The analyses were based upon the modified intent-to-treat population that includes data from participants who were randomized, received at least one dose of study medication (solriamfetol or placebo), and have baseline and at least one post-baseline evaluation.

Demographic characteristics (including gender, age, country of residence, region, race, and ethnicity) were summarized descriptively, along with baseline values of the FOSQ10 and ESS, using frequency and percent for categorical variables and mean, standard deviation (SD), and range for continuous variables.

Distribution-based analyses were used to estimate the minimal detectible change (MDC), which represents the smallest change that can be reliably distinguished from random fluctuation and thus represents the lower bound for estimates of clinically important change. The distribution-based measures that served as estimates of the MDC were 1.0 standard error of measurement (SEM), a value of 0.5 Cohen's $d$ or the standardized effect size [18], and a value of 0.5 Guyatt's statistic (also referred to as the responsiveness statistic) [19].

Next, an anchor-based approach that incorporated either PGI-C or CGI-C (as assessed at week 12) as the anchor was used to determine what magnitude of change on the FOSQ-10 represented a meaningful change from the patient's or clinician's perspective. This type of approach has previously been used for interpreting changes that are meaningful on the ESS and other patient-reported outcomes [20,21].

Spearman correlation coefficients were calculated between each of the anchors (i.e., the impression of change rating) and the change in FOSQ-10 score from baseline to week 12 or early termination. Correlations $\geq 0.30$ in absolute value are recommended to demonstrate suitable anchors and to avoid contamination of interpretation thresholds with noise [22]. Participants were categorized into groups based on their CGI-C and PGI-C ratings, and the change in FOSQ-10 scores from baseline to week 12 or early termination was summarized by category and represented graphically using box plots. Since few participants chose "very much worse," "much worse," or "worse" on the PGI-C and CGI-C, these categories were combined for this analysis.

We evaluated two thresholds for determining a CIR. The first was a PGI-C or CGI-C rating of "minimally improved" or better, and the second was a rating of "much improved" or better. To characterize the association between each specific FOSQ-10 change score and this definition of a CIR, the sensitivity and specificity were calculated, and receiver operating characteristic (ROC) curves were derived using logistic regression analyses. ROC curves simultaneously describe the sensitivity and specificity of a predictive measure as different cutoff values are applied. The optimal values for the FOSQ-10 changes that were best associated with each of the PGI-C or CGI-C threshold values for clinically important change (based on equal importance of sensitivity and specificity) were generated from the corresponding ROC curves. The area under the ROC curve, reported as the C-statistic from the logistic regression model, represents the overall ability of model predictions to discriminate between individuals who do and do not experience clinically important change at the specified level.

\section{Results}

\section{Patient characteristics}

The analysis included a total of 690 participants across the two studies (Table 1). Among participants with narcolepsy $(N=$ 231), $65 \%$ were female, $80 \%$ were white, and $81 \%$ were from North America, and the mean age was 36 years. The mean (SD) ESS score for these participants was 17.2 (3.18), and the mean (SD) FOSQ-10 score was 11.7 (3.03). Among participants with OSA $(N=459), 38 \%$ were female, $76 \%$ were white, and $97 \%$ were from North America, and the mean age was 54 years. OSA participants had a mean (SD) ESS score of 15.2 (3.32) and a mean (SD) FOSQ-10 score of 13.9 (3.01).

\section{Estimating the MDC}

In participants with narcolepsy, the 1.0 SEM, 0.5 Cohen's $d$, and 0.5 Guyatt's statistic were $1.26,1.49$, and 1.03 , respectively. In participants with OSA, the values were 1.48, 1.51, and 1.21 , respectively.

\section{Descriptive analyses}

Spearman correlations between each of the anchors, PGI-C and CGI-C, and changes in FOSQ-10 from baseline to week 12 or early termination were -0.57 and -0.49 for participants with narcolepsy and -0.42 and -0.37 for participants with OSA, confirming that both ratings were suitable for use in anchor-based MID analysis [23]. In general, mean FOSQ-10 change scores were higher in participants whose ratings of change (PGI-C or CGI-C) indicated greater improvement (Table 2 and Fig. 1). 
Table 1 Demographic and baseline characteristics

\begin{tabular}{|c|c|c|}
\hline \multirow[t]{2}{*}{ Characteristic } & \multicolumn{2}{|l|}{ Study } \\
\hline & Narcolepsy & Obstructive sleep apnea \\
\hline Sample size $(N)$ & 231 & 459 \\
\hline \multicolumn{3}{|l|}{ Gender, $n(\%)$} \\
\hline Female & $150(64.9 \%)$ & $172(37.5 \%)$ \\
\hline Male & $81(35.1 \%)$ & $287(62.5 \%)$ \\
\hline \multicolumn{3}{|l|}{ Age, year } \\
\hline Mean & 36.20 & 53.86 \\
\hline SD & 13.15 & 10.96 \\
\hline Range & $18-70$ & $20-75$ \\
\hline \multicolumn{3}{|l|}{ Region, $n(\%)$} \\
\hline Europe & $44(19.0 \%)$ & $15(3.3 \%)$ \\
\hline North America & $187(81.0 \%)$ & $444(96.7 \%)$ \\
\hline \multicolumn{3}{|l|}{ Race, $n(\%)$} \\
\hline American Indian & $2(0.9 \%)$ & $1(0.2 \%)$ \\
\hline Asian & $6(2.6 \%)$ & $17(3.7 \%)$ \\
\hline Black & $33(14.3 \%)$ & $87(19.0 \%)$ \\
\hline Multiple & $5(2.2 \%)$ & $4(0.9 \%)$ \\
\hline Pacific Islander & $1(0.4 \%)$ & $2(0.4 \%)$ \\
\hline White & $184(79.7 \%)$ & $348(75.8 \%)$ \\
\hline \multicolumn{3}{|l|}{ Ethnicity, $n(\%)$} \\
\hline Hispanic & $10(4.3 \%)$ & $40(8.7 \%)$ \\
\hline Not Hispanic & $221(95.7 \%)$ & $419(91.3 \%)$ \\
\hline Mean (SD) FOSQ-10 score & $11.7(3.03)$ & $13.9(3.01)$ \\
\hline Mean (SD) ESS score & $17.2(3.18)$ & $15.2(3.32)$ \\
\hline Mean MWT sleep latency, min ${ }^{a, b}$ & $6.2-8.7^{\mathrm{c}}$ & $12.1-13.6^{\mathrm{c}}$ \\
\hline
\end{tabular}

SD, standard deviation; FOSQ-10, Functional Outcomes of Sleep Questionnaire-10; ESS, Epworth Sleepiness Scale; MWT, Maintenance of Wakefulness Test; OSA, obstructive sleep apnea

${ }^{\text {a }}$ Inclusion criteria included baseline MWT sleep latency $<25 \mathrm{~min}$ for participants with narcolepsy and $<30 \mathrm{~min}$ for participants with OSA

${ }^{\mathrm{b}}$ Narcolepsy, $n=227$ (placebo, $n=57$; combined solriamfetol, $n=170$ ); OSA, $n=450$ (placebo, $n=111$; combined solriamfetol, $n=339$ )

${ }^{\mathrm{c}}$ Range across all treatment groups
The mean changes in FOSQ-10 scores for those who rated themselves (PGI-C) or whose clinician rated them (CGI-C) as "minimally improved" were 2.05 and 1.74 for narcolepsy and 2.08 and 1.66 for OSA. These values exceed the highest estimated value for the MDC, approximately 1.5 in both indications. In participants who were rated as "much improved" on the PGI-C or CGI-C, the mean changes in FOSQ-10 were 4.03 and 4.28 , respectively, for participants with narcolepsy and 3.32 and 3.40 for participants with OSA (Table 2).

\section{Estimating the MID and CIR}

The results of the ROC analyses of the change in FOSQ-10 that was best associated with each definition of improvement (i.e., "minimally improved" or "much improved" or "very much improved" and "much improved" or "very much improved") are presented in Table 3 as empirical estimates of the MID and CIR, respectively, in participants with narcolepsy and OSA. The areas under the ROC curves were also very similar.

The C-statistics (areas under the ROC curve) for the analyses of FOSQ-10 changes associated with PGI-C or CGI-C ratings of "much improved" or "very much improved" were between 0.71 and 0.79 , indicating good accuracy for predicting a true response (Fig. 2 and Fig. 3). For reference, a perfect classifier of responders would have a $\mathrm{C}$-statistic of 1.0; a random classifier would have a value of 0.5 [24]. Sensitivity and specificity were moderate, with values between 0.62 and 0.70 . The optimal FOSQ-10 changes corresponding to a CIR in participants with narcolepsy were 
Table 2 Mean changes in FOSQ-10 by CGI-C and PGI-C ratings

\begin{tabular}{|c|c|c|c|c|c|c|}
\hline \multirow[t]{2}{*}{ Study } & & \multicolumn{5}{|c|}{ Mean change in FOSQ-10 scores ${ }^{\mathrm{a}, \mathrm{b}}$} \\
\hline & & Very much improved & Much improved & Minimally improved & No change & Worse $^{\mathrm{c}}$ \\
\hline \multirow[t]{2}{*}{ 14-002 (Narcolepsy) } & PGI-C & $\begin{array}{l}6.15(2.98) \\
{[n=17]}\end{array}$ & $\begin{array}{l}4.03(2.95) \\
{[n=55]}\end{array}$ & $\begin{array}{l}2.05(2.76) \\
{[n=56]}\end{array}$ & $\begin{array}{l}0.98(2.42) \\
{[n=41]}\end{array}$ & $\begin{array}{l}-0.08(2.05) \\
{[n=22]}\end{array}$ \\
\hline & CGI-C & $\begin{array}{l}4.43(3.00) \\
{[n=18]}\end{array}$ & $\begin{array}{l}4.28(3.00) \\
{[n=58]}\end{array}$ & $\begin{array}{l}1.74(2.85) \\
{[n=60]}\end{array}$ & $\begin{array}{l}0.85(2.35) \\
{[n=45]}\end{array}$ & $\begin{array}{l}0.80(3.25) \\
{[n=14]}\end{array}$ \\
\hline \multirow[t]{2}{*}{$14-003$ (OSA) } & PGI-C & $\begin{array}{l}4.32(3.23) \\
{[n=66]}\end{array}$ & $\begin{array}{l}3.32(2.74) \\
{[n=137]}\end{array}$ & $\begin{array}{l}2.08(2.71) \\
{[n=95]}\end{array}$ & $\begin{array}{l}0.97(2.07) \\
{[n=89]}\end{array}$ & $\begin{array}{l}0.81(2.17) \\
{[n=18]}\end{array}$ \\
\hline & CGI-C & $\begin{array}{l}3.90(3.35) \\
{[n=81]}\end{array}$ & $\begin{array}{l}3.40(2.67) \\
{[n=128]}\end{array}$ & $\begin{array}{l}1.66(2.44) \\
{[n=91]}\end{array}$ & $\begin{array}{l}1.31(2.41) \\
{[n=97]}\end{array}$ & $\begin{array}{l}0.95(2.59) \\
{[n=8]}\end{array}$ \\
\hline
\end{tabular}

FOSQ-10, Functional Outcomes of Sleep Questionnaire-10; CGI-C, Clinician Global Impression of Change; PGI-C, Patient Global Impression of Change; OSA, obstructive sleep apnea

${ }^{a}$ Values in each cell are: mean (SD) $[n]$ FOSQ-10 score

${ }^{\mathrm{b}}$ FOSQ-10 scores range from 5 to 20, with higher scores representing better functioning. Larger change scores represent greater improvement

${ }^{\mathrm{c}}$ Worse indicates minimally worse, much worse, and very much worse, combined due to small sample size

slightly higher than in participants with OSA, 2.5 compared to 2.2. Values obtained with the two anchors were the same, indicating concordance between participants' and clinicians' ratings of change at this level.
In the analyses of FOSQ-10 changes associated with PGI-C or CGI-C ratings of "minimally improved," "much improved," or "very much improved," the C-statistics were between 0.67 and 0.76 (Fig. 2 and Fig. 3). Sensitivity and
Fig. 1 Change in FOSQ-10 by PGI-C rating. FOSQ-10, Functional Outcomes of Sleep Questionnaire-10; PGI-C, Patient Global Impression of Change; mITT, modified intent-to-treat; OSA, obstructive sleep apnea

\section{4-002 (Narcolepsy)}

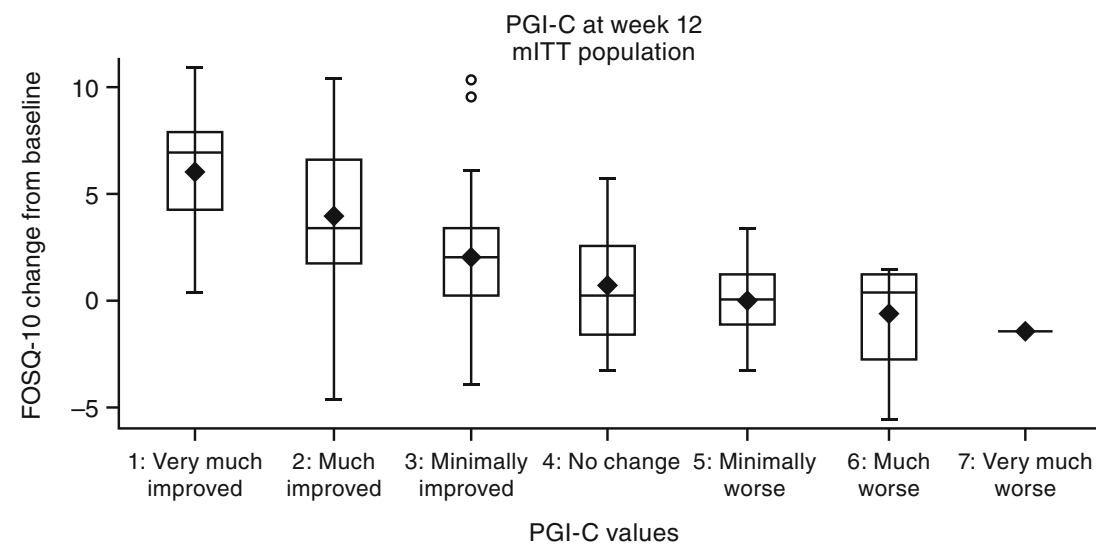

14-003 (OSA)

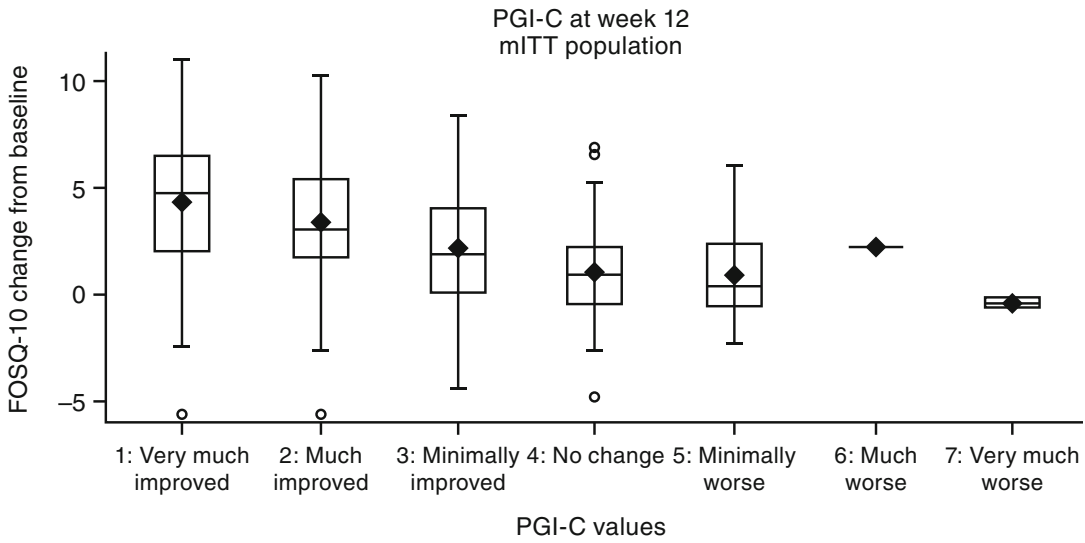


Table 3 FOSQ-10 thresholds from ROC analyses

\begin{tabular}{|c|c|c|c|c|c|c|}
\hline Study & Anchor & Level of change targeted & $\begin{array}{l}\text { Change in } \\
\text { FOSQ-10 scores }\end{array}$ & Sensitivity & Specificity & $\begin{array}{l}\mathrm{C}- \\
\text { statistic }\end{array}$ \\
\hline \multicolumn{7}{|c|}{ To estimate the clinically important response } \\
\hline \multirow[t]{2}{*}{ 14-002 (Narcolepsy) } & PGI-C & Much improved or better & 2.50 & 0.681 & 0.689 & 0.789 \\
\hline & CGI-C & Much improved or better & 2.50 & 0.697 & 0.697 & 0.773 \\
\hline \multirow[t]{2}{*}{$14-003$ (OSA) } & PGI-C & Much improved or better & 2.17 & 0.635 & 0.644 & 0.719 \\
\hline & CGI-C & Much improved or better & 2.17 & 0.622 & 0.643 & 0.711 \\
\hline \multicolumn{7}{|c|}{ To estimate the minimally important difference } \\
\hline \multirow[t]{2}{*}{ 14-002 (Narcolepsy) } & PGI-C & Minimally improved or better & 1.67 & 0.695 & 0.698 & 0.755 \\
\hline & CGI-C & Minimally improved or better & 1.71 & 0.647 & 0.644 & 0.708 \\
\hline \multirow[t]{2}{*}{ 14-003 (OSA) } & PGI-C & Minimally improved or better & 1.83 & 0.681 & 0.682 & 0.724 \\
\hline & CGI-C & Minimally improved or better & 1.88 & 0.623 & 0.638 & 0.672 \\
\hline
\end{tabular}

FOSQ-10, Functional Outcomes of Sleep Questionnaire-10; ROC, receiver operating characteristic; PGI-C, Patient Global Impression of Change; CGIC, Clinician Global Impression of Change; OSA, obstructive sleep apnea

specificity were again moderate, with values between 0.62 and 0.70 . The optimal FOSQ-10 changes corresponding to minimal improvement in participants with narcolepsy and
OSA were more similar, with values of 1.7 for both anchors in narcolepsy and values of 1.8 and 1.9 for PGI-C and CGI$\mathrm{C}$ in participants with OSA.
Fig. 2 ROC curves for PGI-C response $=(\mathbf{a})$ "much improved or better and (b) "minimally improved or better" ROC, receiver operating characteristic; PGI-C, Patient Global Impression of Change; OSA, obstructive sleep apnea

a

14-002 (Narcolepsy)

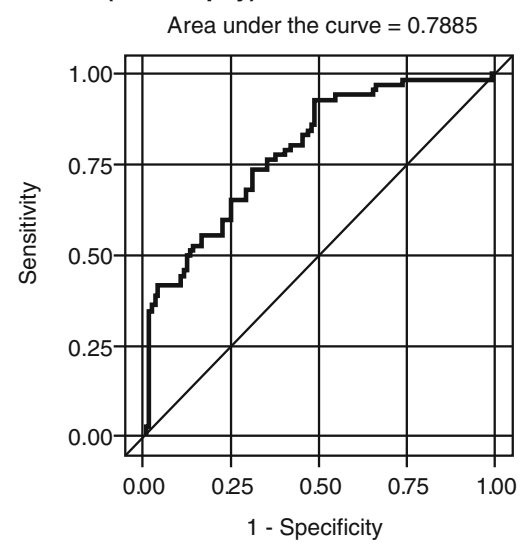

b

14-002 (Narcolepsy)

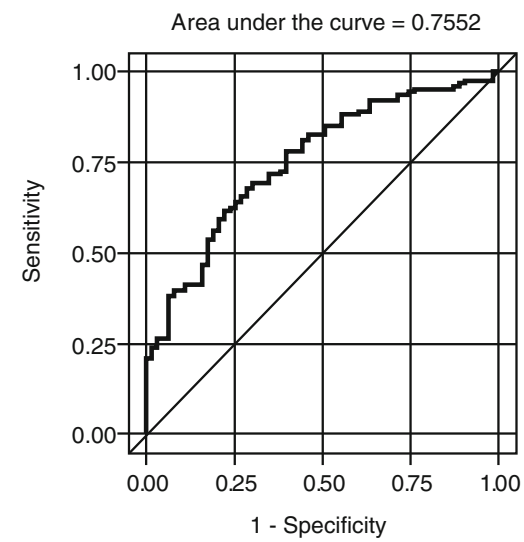

14-003 (OSA)

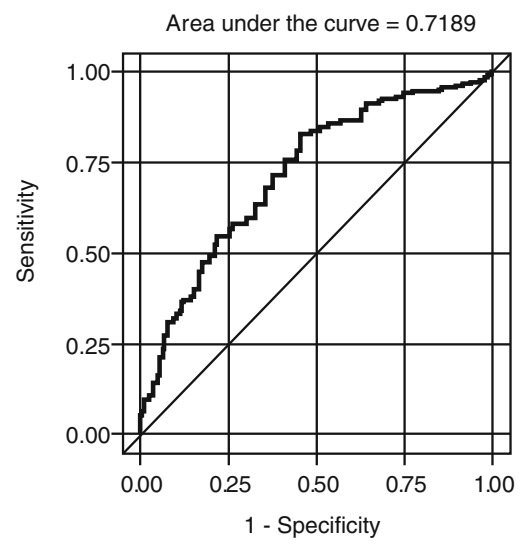

14-003 (OSA)

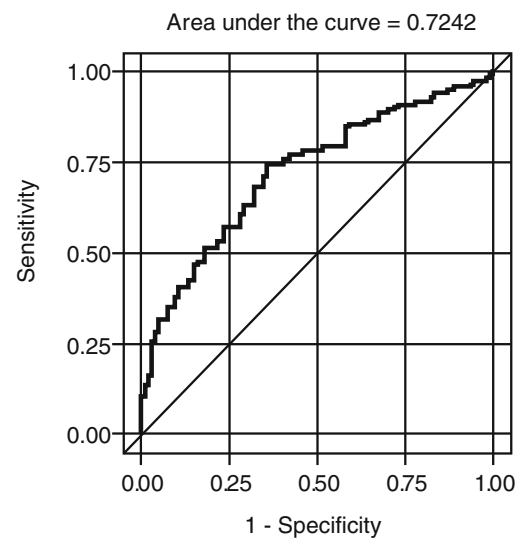


Fig. 3 ROC curves for CGI-C response $=(\mathbf{a})$ "much improved or better" and (b) "minimally improved or better" ROC, receiver operating characteristic; CGI-C, Clinician Global Impression of Change; OSA, obstructive sleep apnea a

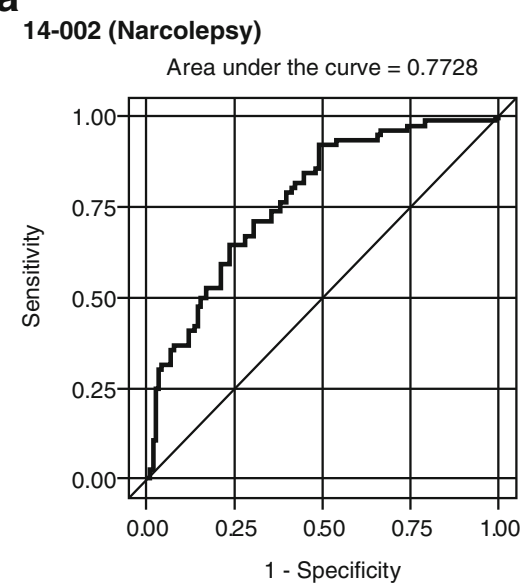

b

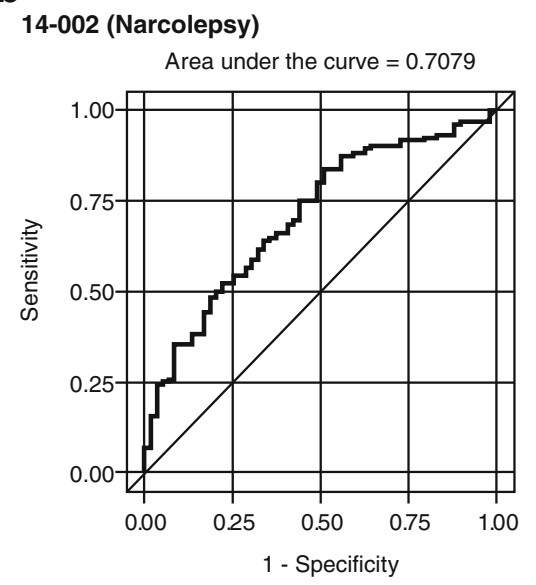

14-003 (OSA)

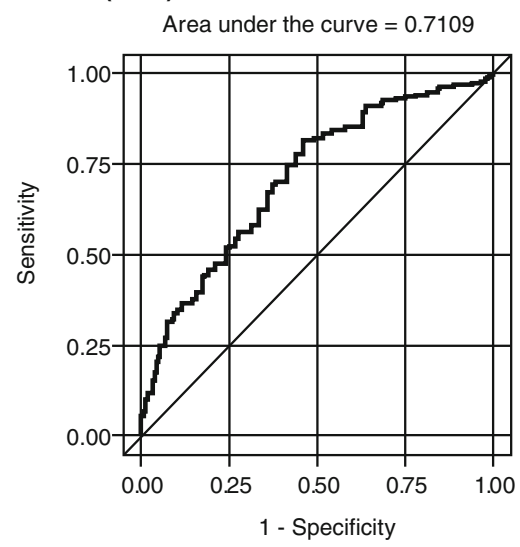

14-003 (OSA)

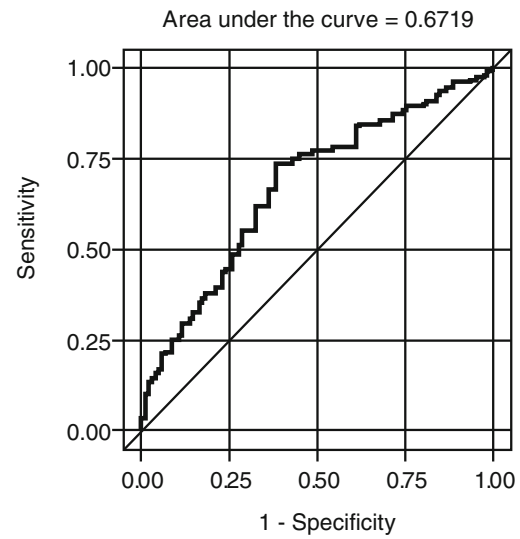

\section{Discussion}

This aim of this study was to provide guidance on how to interpret within-patient changes in the FOSQ-10 over time by estimating thresholds for the MID and CIR. Multiple approaches were employed to provide an estimate of two levels of patient-perceived change in the FOSQ-10 total score: that of minimal change (MID) and the CIR. This work provides clinicians and researchers with benchmarks for evaluating and interpreting FOSQ-10 results in a manner that associates the patient or clinician perception of improvement with the FOSQ-10 total score. To ensure a robust estimate, we used multiple anchors and integrated results from both anchorbased and distribution-based estimates, as well as ROC analyses, in accordance with established recommendations [22].

This study also provided the opportunity to compare levels of change in the FOSQ-10 in participants with narcolepsy and OSA. The MID values for narcolepsy and OSA participants were 1.7 and 1.8 , respectively, and the CIR values for narcolepsy and OSA participants were 2.5 and 2.2 , respectively.

There is increasing recognition that the clinical significance of a change cannot be inferred from statistical significance alone. Determining clinical significance requires an understanding of how the change is perceived and experienced by the patient. The estimation of the MID and CIR provides clinicians and researchers with benchmarks for two levels of change, as perceived by patients, specifically the smallest change that a patient can identify (MID) and that which represents a relevant treatment benefit (CIR). Having both benchmarks available provides the opportunity to evaluate the levels of change of FOSQ-10 scores that may occur over time. The use of many of the anchor-based and distribution-based methods employed in the current study traces back to the early 1990s [23], and these methods have been used individually or together to estimate meaningful change in a variety of diseases, including cancer [25], heart disease [26], chronic obstructive pulmonary disease [27], urinary incontinence [25], inflammatory bowel disease [28], arthritis [29], and asthma [30]. The use of ROC analysis to empirically determine cutoff values has been utilized in previous studies to establish a CIR [13], including in studies of narcolepsy using the PGI-C or CGI-C as anchors [20, 21].

These analyses have several notable strengths. First, the analyses incorporated both distribution- and anchor-based methods. Two anchors, one based on a PGI-C and the other on a CGI-C, were used. Notably, the anchors reflected similar 
mean changes in FOSQ-10 scores, thereby strengthening the interpretation of what changes in FOSQ-10 scores represent. Additionally, the estimation of the MID and CIR was derived in two distinct patient populations with EDS. However, a limitation is that few participants in the current study experienced a worsening in their condition, precluding the estimation of either an MID or a CIR for worsening symptoms. This is consistent with results previously reported from these studies that indicated improvements in EDS with solriamfetol treatment, as demonstrated by least square mean decreases in ESS scores (narcolepsy, -3.8 to -6.4 ; OSA, -5.0 to -7.9 ) and increases in mean MWT sleep latency (narcolepsy, +4.7 to + $12.3 \mathrm{~min}$; OSA, +4.7 to $+13.0 \mathrm{~min}$ ) from baseline to week 12 relative to placebo (ESS scores: narcolepsy, - 1.6; OSA, 3.3; MWT sleep latency: narcolepsy, $2.1 \mathrm{~min}$; OSA, $0.2 \mathrm{~min}$ ) $[14,15]$. This is also consistent with recently published data from the same studies used in this analysis that demonstrated moderate to high correlations between changes in FOSQ-10 and ESS scores or mean MWT sleep latency [31]. Additionally, we note that the data presented here were obtained in the context of double-blind, placebo-controlled, randomized, parallel-group, phase 3 studies evaluating the effects of solriamfetol in the treatment of EDS. It is unclear to what extent the current findings are generalizable to other patient populations with EDS or with other therapies.

Despite these limitations, the CIR estimate for the FOSQ10 overall score of 2.2 to 2.5 for adult populations with OSA or narcolepsy treated for EDS will be valuable for interpreting changes over time and defining a clinical responder. Future research in other populations or those receiving other therapies for EDS will add to this work.

Acknowledgments Preliminary findings from this study were presented at the 32nd Annual Meeting of the Associated Professional Sleep Societies (APSS), June 2-6, 2018, Baltimore, MD.

Authors' contributions All authors collaborated in the preparation of the manuscript and critically reviewed and provided revisions to the paper. All authors had access to the data and assume responsibility for the completeness and accuracy of the data and data analyses.

Funding This study was funded by Jazz Pharmaceuticals. Editorial assistance in formatting, proofreading, and copyediting was provided by Peloton Advantage and was funded by Jazz Pharmaceuticals.

Data availability All relevant data are provided within the manuscript and supporting files.

\section{Compliance with ethical standards}

Conflict of interest TEW has received royalty fees for the use of the FOSQ-10 from Jazz Pharmaceuticals, ResMed, Bayer AD, Cook Medical, Nyxoah, RWS, Merck \& Co. Inc., Verily Life Science, WCG MedAvante Prophase, Stratevi, Evidation Health, and Philips Respironics. RDC is a consultant to Health Outcomes Solutions, which received funding from Jazz Pharmaceuticals for conducting these analyses. DMM, MB, and SM are employees of Jazz Pharmaceuticals who, in the course of their employment, have received stock options exercisable for, and other stock awards of, ordinary shares of Jazz Pharmaceuticals, LLC. SDM is an employee of Health Outcomes Solutions, which received funding from Jazz Pharmaceuticals for conducting these analyses.

Ethics approval These studies were conducted in line with the principles of the Declaration of Helsinki and were approved by institutional review board or ethics committees at each site.

Consent to participate All participants provided written informed consent.

Consent for publication All authors granted final approval of the manuscript for submission.

Code availability Not applicable.

Open Access This article is licensed under a Creative Commons Attribution 4.0 International License, which permits use, sharing, adaptation, distribution and reproduction in any medium or format, as long as you give appropriate credit to the original author(s) and the source, provide a link to the Creative Commons licence, and indicate if changes were made. The images or other third party material in this article are included in the article's Creative Commons licence, unless indicated otherwise in a credit line to the material. If material is not included in the article's Creative Commons licence and your intended use is not permitted by statutory regulation or exceeds the permitted use, you will need to obtain permission directly from the copyright holder. To view a copy of this licence, visit http://creativecommons.org/licenses/by/4.0/.

\section{References}

1. Fernandez-Mendoza J, Vgontzas AN, Kritikou I, Calhoun SL, Liao D, Bixler EO (2015) Natural history of excessive daytime sleepiness: role of obesity, weight loss, depression, and sleep propensity. Sleep 38(3):351-360. https://doi.org/10.5665/sleep.4488

2. Zhang J, Han F (2017) Sleepiness in narcolepsy. Sleep Med Clin 12(3):323-330. https://doi.org/10.1016/j.jsmc.2017.03.008

3. Young T, Peppard PE, Gottlieb DJ (2002) Epidemiology of obstructive sleep apnea: a population health perspective. Am J Respir Crit Care Med 165(9):1217-1239

4. Young T, Palta M, Dempsey J, Skatrud J, Weber S, Badr S (1993) The occurrence of sleep-disordered breathing among middle-aged adults. N Engl J Med 328(17):1230-1235. https://doi.org/10.1056/ NEJM199304293281704

5. Fu Y, Xu H, Xia Y, Qian Y, Li X, Zou J, Wang Y, Meng L, Tang X, Zhu H, Zhou H, Su K, Yu D, Yi H, Guan J, Yin S (2017) Excessive daytime sleepiness and metabolic syndrome in men with obstructive sleep apnea: a large cross-sectional study. Oncotarget 8(45): 79693-79702. https://doi.org/10.18632/oncotarget.19113

6. Gasa M, Tamisier R, Launois SH, Sapene M, Martin F, Stach B, Grillet Y, Levy P, Pepin JL, Scientific Council of the Sleep Registry of the French Federation of Pneumology-FFP (2013) Residual sleepiness in sleep apnea patients treated by continuous positive airway pressure. J Sleep Res 22(4):389-397. https://doi.org/10. $1111 /$ jsr.12039

7. Dean B, Aguilar D, Shapiro C, Orr WC, Isserman JA, Calimlim B, Rippon GA (2010) Impaired health status, daily functioning, and work productivity in adults with excessive sleepiness. J Occup 
Environ Med 52(2):144-149. https://doi.org/10.1097/JOM. 0b013e3181c99505

8. Bioulac S, Micoulaud-Franchi JA, Arnaud M, Sagaspe P, Moore N, Salvo F, Philip P (2017) Risk of motor vehicle accidents related to sleepiness at the wheel: a systematic review and meta-analysis. Sleep 40(10). https://doi.org/10.1093/sleep/zsx134

9. Weaver TE, Laizner AM, Evans LK, Maislin G, Chugh DK, Lyon K, Smith PL, Schwartz AR, Redline S, Pack AI, Dinges DF (1997) An instrument to measure functional status outcomes for disorders of excessive sleepiness. Sleep 20(10):835-843

10. Chasens ER, Ratcliffe SJ, Weaver TE (2009) Development of the FOSQ-10: a short version of the functional outcomes of sleep questionnaire. Sleep 32(7):915-919

11. Brozek JL, Guyatt GH, Schunemann HJ (2006) How a wellgrounded minimal important difference can enhance transparency of labelling claims and improve interpretation of a patient reported outcome measure. Health Qual Life Outcomes 4:69. https://doi.org/ 10.1186/1477-7525-4-69

12. Schunemann HJ, Akl EA, Guyatt GH (2006) Interpreting the results of patient reported outcome measures in clinical trials: the clinician's perspective. Health Qual Life Outcomes 4:62. https://doi. org/10.1186/1477-7525-4-62

13. Coon CD, Cappelleri JC (2016) Interpreting change in scores on patient-reported outcome instruments. Ther Innov Regul Sci 50(1): 22-29. https://doi.org/10.1177/2168479015622667

14. Thorpy MJ, Shapiro C, Mayer G, Corser BC, Emsellem H, Plazzi G, Chen D, Carter LP, Wang H, Lu Y, Black J, Dauvilliers Y (2019) A randomized study of solriamfetol for excessive sleepiness in narcolepsy. Ann Neurol 85(3):359-370. https://doi.org/10.1002/ ana. 25423

15. Schweitzer PK, Rosenberg R, Zammit GK, Gotfried M, Chen D, Carter LP, Wang H, Lu Y, Black J, Malhotra A, Strohl KP (2019) Solriamfetol for excessive sleepiness in obstructive sleep apnea (TONES 3): a randomized controlled trial. Am J Respir Crit Care Med 199(11):1421-1431. https://doi.org/10.1164/rccm.201806$1100 \mathrm{OC}$

16. Mitler MM, Gujavarty KS, Browman CP (1982) Maintenance of wakefulness test: a polysomnographic technique for evaluation treatment efficacy in patients with excessive somnolence. Electroencephalogr Clin Neurophysiol 53(6):658-661

17. Johns MW (1991) A new method for measuring daytime sleepiness: the Epworth sleepiness scale. Sleep 14(6):540-545

18. Cohen J (1988) Statistical power analysis for the behavioral sciences, 2nd edn. Lawrence Erlbaum, Hillsdale, NJ

19. Guyatt GH, Bombardier C, Tugwell PX (1986) Measuring diseasespecific quality of life in clinical trials. CMAJ 134(8):889-895

20. Scrima L, Emsellem HA, Becker PM, Ruoff C, Lankford A, Bream G, Khayrallah M, Lu Y, Black J (2017) Identifying clinically important difference on the Epworth sleepiness scale: results from a narcolepsy clinical trial of JZP-110. Sleep Med 38:108-112. https:// doi.org/10.1016/j.sleep.2017.07.006

21. Steffen AD, Lai C, Weaver TE (2018) Criteria for gauging response to sodium oxybate for narcolepsy. J Sleep Res 27(4):e12628. https://doi.org/10.1111/jsr.12628

22. Crosby RD, Kolotkin RL, Williams GR (2003) Defining clinically meaningful change in health-related quality of life. J Clin Epidemiol 56(5):395-407

23. Lydick E, Epstein RS (1993) Interpretation of quality of life changes. Qual Life Res 2(3):221-226

24. Hanley JA (1989) Receiver operating characteristic (ROC) methodology: the state of the art. Crit Rev Diagn Imaging 29(3):307335

25. Patrick DL, Gagnon DD, Zagari MJ, Mathijs R, Sweetenham J (2003) Assessing the clinical significance of health-related quality of life (HrQOL) improvements in anaemic cancer patients receiving epoetin alfa. Eur J Cancer 39(3):335-345. https://doi.org/10.1016/ s0959-8049(02)00628-7

26. Wyrwich KW, Nienaber NA, Tierney WM, Wolinsky FD (1999) Linking clinical relevance and statistical significance in evaluating intra-individual changes in health-related quality of life. Med Care 37(5):469-478

27. Wyrwich KW, Tierney WM, Wolinsky FD (1999) Further evidence supporting an SEM-based criterion for identifying meaningful intra-individual changes in health-related quality of life. J Clin Epidemiol 52(9):861-873

28. Jowett SL, Seal CJ, Barton JR, Welfare MR (2001) The short inflammatory bowel disease questionnaire is reliable and responsive to clinically important change in ulcerative colitis. Am J Gastroenterol 96(10):2921-2928. https://doi.org/10.1111/j.15720241.2001.04682.x

29. Kosinski M, Zhao SZ, Dedhiya S, Osterhaus JT, Ware JE Jr (2000) Determining minimally important changes in generic and diseasespecific health-related quality of life questionnaires in clinical trials of rheumatoid arthritis. Arthritis Rheum 43(7):1478-1487

30. Wyrwich KW, Tierney WM, Wolinsky FD (2002) Using the standard error of measurement to identify important changes on the asthma quality of life questionnaire. Qual Life Res 11(1):1-7

31. Weaver TE, Mathias SD, Crosby RD, Bron M, Bujanover S, Menno D, Villa KF, Drake C (2020) Relationship between sleep efficacy endpoints and measures of functional status and healthrelated quality of life in participants with narcolepsy or obstructive sleep apnea treated for excessive daytime sleepiness. J Sleep Res: e13210. https://doi.org/10.1111/jsr.13210

Publisher's note Springer Nature remains neutral with regard to jurisdictional claims in published maps and institutional affiliations. 